\title{
Convergence results for a common solution of a finite family of variational inequality problems for monotone mappings with Bregman distance function
}

\author{
Naseer Shahzad ${ }^{1 *}$, Habtu Zegeye ${ }^{2}$ and Abdullah Alotaibi ${ }^{1}$
}

${ }^{*}$ Correspondence:

nshahzad@kau.edu.sa

1 Department of Mathematics, King

Abdulaziz University, P.O. Box 80203

Jeddah, 21589, Saudi Arabia

Full list of author information is

available at the end of the article

\begin{abstract}
In this paper, we introduce an iterative process which converges strongly to a common solution of a finite family of variational inequality problems for monotone mappings with Bregman distance function. Our convergence theorem is applied to the convex minimization problem. Our theorems extend and unify most of the results that have been proved for the class of monotone mappings.

MSC: 47H05; 47J05; 47J25
\end{abstract}

Keywords: Bregman projection; monotone mappings; strong convergence; variational inequality problems

\section{Introduction}

Throughout this paper, $E$ is a real reflexive Banach space with $E^{*}$ as its dual and $f: E \rightarrow$ $(-\infty,+\infty]$ is a proper, lower semicontinuous and convex function. We denote by $\operatorname{dom} f$ the domain of $f$, defined by $\operatorname{dom} f:=\{x \in E: f(x)<+\infty\}$. For any $x \in \operatorname{int}(\operatorname{dom} f)$ and $y \in E$, the right-hand derivative of $f$ at $x$ in the direction of $y$ is defined by

$$
f^{0}(x, y):=\lim _{t \rightarrow 0^{+}} \frac{f(x+t y)-f(x)}{t} .
$$

The function $f$ is said to be Gâteaux differentiable at $x$ if $\lim _{t \rightarrow 0^{+}}(f(x+t y)-f(x)) / t$ exists for any $y \in E$. In this case, $f^{0}(x, y)$ coincides with $\nabla f(x)$, the value of the gradient $\nabla f$ of $f$ at $x$. The function $f$ is said to be Gâteaux differentiable if it is Gâteaux differentiable for any $x \in \operatorname{int}(\operatorname{dom} f)$. The function $f$ is said to be Fréchet differentiable at $x$ if this limit is attained uniformly in $\|y\|=1$. We say that $f$ is uniformly Fréchet differentiable on a subset $C$ of $E$ if the limit is attained uniformly for $x \in C$ and $\|y\|=1$.

Let $f: E \rightarrow(-\infty,+\infty]$ be a Gâteaux differentiable function. The function $D_{f}: \operatorname{dom} f \times$ $\operatorname{int}(\operatorname{dom} f) \rightarrow[0,+\infty)$ defined by

$$
D_{f}(x, y):=f(x)-f(y)-\langle\nabla f(y), x-y\rangle
$$

is called the Bregman distance with respect to $f$ [1].

@2013 Shahzad et al: licensee Springer. This is an Open Access article distributed under the terms of the Creative Commons Attribution License (http://creativecommons.org/licenses/by/2.0), which permits unrestricted use, distribution, and reproduction in any medium, provided the original work is properly cited. 
A Bregman projection with respect to $f[1]$ of $x \in \operatorname{int}(\operatorname{dom} f)$ onto the nonempty, closed and convex set $C \subset \operatorname{int}(\operatorname{dom} f)$ is the unique vector $P_{C}^{f}(x) \in C$ satisfying

$$
D_{f}\left(P_{C}^{f}(x), x\right)=\inf \left\{D_{f}(y, x): y \in C\right\} .
$$

Remark 1.1 If $E$ is a smooth and strictly convex Banach space and $f(x)=\|x\|^{2}$ for all $x \in E$, then we have that $\nabla f(x)=2 J(x)$ for all $x \in E$, where $J$ the normalized duality mapping from $E$ into $E^{*}$, and hence $D_{f}(x, y)$ becomes $D_{f}(x, y)=\|x\|^{2}-2\langle x, J y\rangle+\|y\|^{2}$ for all $x, y \in E$, which is the Lyapunov function introduced by Alber [2] and studied by many authors (see, e.g., [3-9] and the references therein). In addition, under the same condition, the Bregman projection $P_{C}^{f}(x)$ reduces to the generalized projection $\Pi_{C}(x)$ (see, e.g., [2]) which is defined by

$$
\phi\left(\Pi_{C}(x), x\right)=\min _{y \in C} \phi(y, x) .
$$

If $E=H$, a Hilbert space, $J$ is the identity mapping, and hence the Bregman projection $P_{C}^{f}(x)$ reduces to the metric projection of $H$ on to $C, P_{C}(x)$.

A mapping $A: D(A) \subset E \rightarrow E^{*}$ is said to be $\gamma$-inverse strongly monotone if there exists a positive real number $\gamma$ such that

$$
\langle A x-A y, x-y\rangle \geq \gamma\|A x-A y\|^{2} \quad \text { for all } x, y \in D(A) .
$$

$A$ is said to be monotone if, for each $x, y \in D(A)$, the following inequality holds:

$$
\langle A x-A y, x-y\rangle \geq 0 .
$$

Clearly, the class of monotone mappings includes the class of $\gamma$-inverse strongly monotone mappings.

Let $C$ be a nonempty, closed and convex subset of $E$ and $A: C \rightarrow E^{*}$ be a monotone mapping. The problem of finding

a point $u \in C$ such that $\langle A u, v-u\rangle \geq 0$ for all $v \in C$

is called the variational inequality problem. The set of solutions of the variational inequality is denoted by $V I(C, A)$.

Variational inequality problems are related with the convex minimization problem, the zero of monotone mappings and the complementarity problem. Consequently, many researchers (see, e.g., $[3,5,10-15])$ have made efforts to obtain iterative methods for approximating solutions of variational inequality problems.

If $E=H$, a Hilbert space, Iiduka et al. [16] introduced the following projection algorithm:

$$
x_{1}=x \in C, \quad x_{n+1}=P_{C}\left(x_{n}-\alpha_{n} A x_{n}\right) \quad \text { for any } n \geq 1,
$$

where $P_{C}$ is the metric projection from $H$ onto $C$ and $\left\{\alpha_{n}\right\}$ is a sequence of positive real numbers. They proved that the sequence $\left\{x_{n}\right\}$ generated by (1.6) converges weakly to some element of $V I(C, A)$ provided that $A$ is a $\gamma$-inverse strongly monotone mapping. 
If $E$ is a 2-uniformly convex and uniformly smooth Banach space, and $A$ is $\gamma$-inverse strongly monotone, Iiduka and Takahashi [17] introduced the following iteration scheme for finding a solution of the variational inequality problem:

$$
x_{n+1}=\Pi_{C} J^{-1}\left(J x_{n}-\alpha_{n} A x_{n}\right) \quad \text { for any } n \geq 1 \text {, }
$$

where $\Pi_{C}$ is the generalized projection from $E$ onto $C, J$ is the normalized duality mapping from $E$ into $E^{*}$ and $\left\{\alpha_{n}\right\}$ is a sequence of positive real numbers. They proved that the sequence $\left\{x_{n}\right\}$ generated by (1.7) converges weakly to some element of $V I(C, A)$.

It is worth mentioning that the convergence obtained above is weak convergence. Our concern now is to look for an iteration scheme which converges strongly to a solution of the variational inequality problem for a monotone mapping $A$.

In this regard, when $E$ is a 2-uniformly convex and uniformly smooth Banach space and $A$ is a $\gamma$-inverse strongly monotone mapping satisfying $\|A u\| \leq\|A y-A u\|$ for all $y \in C$ and $u \in V I(C, A)$ for $V I(C, A) \neq \emptyset$, Iiduka and Takahashi [10] studied the following iterative scheme for a solution of the variational inequality problem:

$$
\left\{\begin{array}{l}
x_{0} \in K \quad \text { chosen arbitrarily, } \\
y_{n}=\Pi_{C} J^{-1}\left(J x_{n}-\alpha_{n} A x_{n}\right), \\
C_{n}=\left\{z \in E: \phi\left(z, y_{n}\right) \leq \phi\left(z, x_{n}\right)\right\} \\
Q_{n}=\left\{z \in E:\left\langle x_{n}-z, J x_{0}-J x_{n}\right\rangle \geq 0\right\} \\
x_{n+1}=\Pi_{C_{n} \cap Q_{n}}\left(x_{0}\right), \quad n \geq 1,
\end{array}\right.
$$

where $\left\{\alpha_{n}\right\}$ is a positive real sequence satisfying certain mild conditions and $\Pi_{C_{n} \cap Q_{n}}$ is the generalized projection from $E$ onto $C_{n} \cap Q_{n}, J$ is the duality mapping from $E$ into $E^{*}$. Then they proved that the sequence $\left\{x_{n}\right\}$ converges strongly to an element of $\operatorname{VI}(C, A)$.

Recently, Zegeye and Shahzad [18] studied the following iterative scheme for a common point of a solution of two variational inequality problems for continuous monotone mappings in a uniformly smooth and strictly convex real Banach space $E$ which also enjoys the Kadec-Klee property:

$$
\left\{\begin{array}{l}
x_{0} \in C_{0}=C \quad \text { chosen arbitrarily, } \\
u_{n}=T_{1, \gamma_{n}} x_{n} ; \quad v_{n}=T_{2, \gamma_{n}} x_{n}, \\
w_{n}=J^{-1}\left(\beta J u_{n}+(1-\beta) J J_{n}\right) \\
C_{n+1}=\left\{z \in C_{n}: \phi\left(z, w_{n}\right) \leq \phi\left(z, x_{n}\right)\right\} \\
x_{n+1}=\prod_{C_{n+1}}\left(x_{0}\right), \quad n \geq 0,
\end{array}\right.
$$

where $T_{i, \gamma} x:=\left\{z \in C:\left\langle A_{i} z, y-z\right\rangle+\frac{1}{\gamma}\langle y-z, J z-J x\rangle \geq 0, \forall y \in C\right\}$ for all $x \in E, i=1,2$, and $\beta, \gamma_{n} \in(0,1)$ satisfy certain mild conditions. Then they proved that the sequence $\left\{x_{n}\right\}$ converges strongly to $\Pi_{F}\left(x_{0}\right)$, where $\Pi_{F}$ is the generalized projection from $E$ onto $F:=\bigcap_{i=1}^{2} V I\left(C, A_{i}\right) \neq \emptyset$.

In 1967, Bregman [1] discovered an elegant and effective technique for using the socalled Bregman distance function $D_{f}(\cdot, \cdot)$ in the process of designing and analyzing feasibility and optimization algorithms. Using Bregman's distance function and its properties, authors have opened a growing area of research not only for iterative algorithms of solving 
feasibility and optimization problems but also for algorithms of solving nonlinear, equilibrium, variational inequality, fixed point problems and others (see, e.g., [19-25] and the references therein).

In 2010, Reich and Sabach [25] proposed an algorithm for finding a common zero point of a finite family of maximal monotone mappings $A_{i}: E \rightarrow 2^{E^{*}}(i=1,2, \ldots, N)$ in a general reflexive Banach space $E$ as follows:

$$
\left\{\begin{array}{l}
x_{0} \in E \quad \text { chosen arbitrarily, } \\
y_{n}^{i}=\operatorname{Res}_{\lambda_{n}^{i} A_{i}}\left(x_{n}+e_{n}^{i}\right) \\
C_{n}^{i}=\left\{z \in E: D_{f}\left(z, y_{n}^{i}\right) \leq D_{f}\left(z, x_{n}+e_{n}^{i}\right)\right\}, \\
C_{n}=\bigcap_{i=1}^{N} C_{n}^{i}, \\
Q_{n}^{i}=\left\{z \in E:\left\langle\nabla f\left(x_{0}\right)-\nabla f\left(x_{n}\right), z-x_{n}\right\rangle \leq 0\right\}, \\
x_{n+1}=P_{C_{n} \cap Q_{n}}^{f}\left(x_{0}\right), \quad \forall n \geq 0,
\end{array}\right.
$$

where $\left\{\lambda_{n}^{i}\right\}_{i=1}^{N} \subset(0, \infty),\left\{e_{n}^{i}\right\}_{i=1}^{N}$ are error sequences in $E$ with $e_{n}^{i} \rightarrow 0$ and $P_{C}^{f}$ is the Bregman projection with respect to $f$ from $E$ onto a closed and convex subset $C$ of $E$. Those authors showed that the sequence $\left\{x_{n}\right\}$ defined by (1.10) converges strongly to a common element in $\bigcap_{i=1}^{N} A^{-1}\left(0^{*}\right)=\bigcap_{i=1}^{N} V I\left(E, A_{i}\right)$ under some mild conditions. Similar results are also available in $[26,27]$.

Remark 1.2 But it is worth mentioning that the iteration processes (1.8)-(1.10) seem difficult in the sense that at each stage of iteration, the set(s) $C_{n}$ and (or) $Q_{n}$ is (are) computed and the next iterate is taken as the Bregman projection of $x_{0}$ onto the intersection of $C_{n}$ and $Q_{n}$ (or $Q_{n}$ ). This seems difficult to do in applications.

It is our purpose in this paper to introduce an iterative scheme $\left\{x_{n}\right\}$ which converges strongly to a common solution of a finite family of variational inequality problems for monotone mappings in real reflexive Banach spaces. Our scheme does not involve computations of $C_{n}$ or $Q_{n}$ for each $n \geq 1$. Furthermore, we apply our convergence theorem to a convex minimization problem. Our theorems extend and unify most of the results that have been proved for this important class of nonlinear operators.

\section{Preliminaries}

Let $x \in \operatorname{int}(\operatorname{dom} f)$. The subdifferential of $f$ at $x$ is the convex set defined by $\partial f(x)=\left\{x^{*} \in\right.$ $\left.E^{*}: f(x)+\left\langle x^{*}, y-x\right\rangle \leq f(y), \forall y \in E\right\}$, where the Fenchel conjugate of $f$ is the function $f^{*}$ : $E^{*} \rightarrow(-\infty,+\infty]$ defined by $f^{*}\left(x^{*}\right)=\sup \left\{\left\langle x^{*}, x\right\rangle-f(x): x \in E\right\}$.

The function $f$ is said to be:

(i) Essentially smooth if $\partial f$ is both locally bounded and single-valued on its domain.

(ii) Essentially strictly convex if $(\partial f)^{-1}$ is locally bounded on its domain and $f$ is strictly convex on every convex subset of $\operatorname{dom} f$.

(iii) Legendre if it is both essentially smooth and essentially strictly convex.

We remark that we have the following:

(i) $f$ is essentially smooth if and only if $f^{*}$ is essentially strictly convex (see [19], Theorem 5.4).

(ii) $(\partial f)^{-1}=\partial f^{*}($ see $[28])$.

(iii) $f$ is Legendre if and only if $f^{*}$ is Legendre (see [19], Corollary 5.5). 
(iv) If $f$ is Legendre, then $\nabla f$ is a bijection satisfying $\nabla f=\left(\nabla f^{*}\right)^{-1}$,

$\operatorname{ran} \nabla f=\operatorname{dom} \nabla f^{*}=\operatorname{int}\left(\operatorname{dom} f^{*}\right)$ and $\operatorname{ran} \nabla f^{*}=\operatorname{dom} \nabla f=\operatorname{int}(\operatorname{dom} f)$ (see [19],

Theorem 5.10).

When the subdifferential of $f$ is single-valued, then $\partial f=\nabla f$ (see [29]).

A function $f$ on $E$ is coercive [30] if the sublevel set of $f$ is bounded; equivalently, $\lim _{\|x\| \rightarrow \infty} f(x)=\infty$.

Let $f: E \rightarrow(-\infty,+\infty]$ be a convex and Gâteaux differentiable function. The modulus of total convexity of $f$ at $x \in \operatorname{dom} f$ is the function $v_{f}(x, \cdot):[0,+\infty) \rightarrow[0,+\infty]$ defined by

$$
v_{f}(x, t):=\inf \left\{D_{f}(y, x): y \in \operatorname{dom} f,\|y-x\|=t\right\} .
$$

The function $f$ is called totally convex at $x$ if $v_{f}(x, t)>0$, whenever $t>0$. The function $f$ is called totally convex if it is totally convex at any point $x \in \operatorname{int}(\operatorname{dom} f)$ and it is said to be totally convex on bounded sets if $v_{f}(B, t)>0$ for any nonempty bounded subset $B$ of $E$ and $t>0$, where the modulus of total convexity of the function $f$ on the set $B$ is the function $v_{f}: \operatorname{int}(\operatorname{dom} f) \times[0,+\infty) \rightarrow[0,+\infty]$ defined by

$$
v_{f}(B, t):=\inf \left\{V_{f}(x, t): x \in B \cap \operatorname{dom} f\right\} .
$$

We know that $f$ is totally convex on bounded sets if and only if $f$ is uniformly convex on bounded sets (see [22], Theorem 2.10). The following lemmas will be useful in the proof of our main result.

Lemma 2.1 [31] The function $f: E \rightarrow(-\infty,+\infty$ ] is totally convex on bounded subsets of $E$ if and only iffor any two sequences $\left\{x_{n}\right\}$ and $\left\{y_{n}\right\}$ in $\operatorname{int}(\operatorname{dom} f)$ and $\operatorname{dom} f$, respectively, such that the first one is bounded,

$$
\lim _{n \rightarrow \infty} D_{f}\left(y_{n}, x_{n}\right)=0 \quad \Longrightarrow \quad \lim _{n \rightarrow \infty}\left\|y_{n}-x_{n}\right\|=0 .
$$

Lemma 2.2 [22] Let C be a nonempty, closed and convex subset of E. Let $f: E \rightarrow(-\infty,+\infty$ ] be a Gâteaux differentiable and totally convex function, and let $x \in E$. Then:

(i) $z=P_{C}^{f}(x)$ if and only if $\langle\nabla f(x)-\nabla f(z), y-z\rangle \leq 0, \forall y \in C$.

(ii) $D_{f}\left(y, P_{C}^{f}(x)\right)+D_{f}\left(P_{C}^{f}(x), x\right) \leq D_{f}(y, x), \forall y \in C$.

Lemma 2.3 [29] Let $f: E \rightarrow(-\infty,+\infty$ ] be a proper, lower semi-continuous and convex function, then $f^{*}: E^{*} \rightarrow(-\infty,+\infty]$ is a proper, weak ${ }^{*}$ lower semicontinuous and convex function. Thus, for all $z \in E$, we have

$$
D_{f}\left(z, \nabla f^{*}\left(\sum_{i=1}^{N} t_{i} \nabla f\left(x_{i}\right)\right)\right) \leq \sum_{i=1}^{N} t_{i} D_{f}\left(z, x_{i}\right) .
$$

Lemma 2.4 [32] Let $f: E \rightarrow \mathbb{R}$ be Gâteaux differentiable on $\operatorname{int}(\operatorname{dom} f)$ such that $\nabla f^{*}$ is bounded on bounded subsets of $\operatorname{dom} f^{*}$. Let $x^{*} \in X$ and $\left\{x_{n}\right\} \subset E$. If $\left\{D_{f}\left(x, x_{n}\right)\right\}$ is bounded, so is the sequence $\left\{x_{n}\right\}$.

Let $f: E \rightarrow \mathbb{R}$ be a Legendre and Gâteaux differentiable function. Following [2] and [33], we make use of the function $V_{f}: E \times E^{*} \rightarrow[0,+\infty)$ associated with $f$, which is defined by

$$
V_{f}\left(x, x^{*}\right)=f(x)-\left\langle x, x^{*}\right\rangle+f^{*}\left(x^{*}\right), \quad \forall x \in E, x^{*} \in E^{*} .
$$


Then $V_{f}$ is nonnegative and

$$
V_{f}\left(x, x^{*}\right)=D_{f}\left(x, \nabla f^{*}\left(x^{*}\right)\right) \quad \text { for all } x \in E \text { and } x^{*} \in E^{*} \text {. }
$$

Moreover, by the subdifferential inequality,

$$
V_{f}\left(x, x^{*}\right)+\left\langle y^{*}, \nabla f^{*}\left(x^{*}\right)-x\right\rangle \leq V_{f}\left(x, x^{*}+y^{*}\right),
$$

$\forall x \in E$ and $x^{*}, y^{*} \in E^{*}($ see $[34])$

Lemma 2.5 [35] Let $\left\{a_{n}\right\}$ be a sequence of nonnegative real numbers satisfying the following relation:

$$
a_{n+1} \leq\left(1-\alpha_{n}\right) a_{n}+\alpha_{n} \delta_{n}, \quad n \geq n_{0},
$$

where $\left\{\alpha_{n}\right\} \subset(0,1)$ and $\left\{\delta_{n}\right\} \subset \mathbb{R}$ satisfy the following conditions: $\lim _{n \rightarrow \infty} \alpha_{n}=0, \sum_{n=1}^{\infty} \alpha_{n}=$ $\infty$, and $\limsup _{n \rightarrow \infty} \delta_{n} \leq 0$. Then $\lim _{n \rightarrow \infty} a_{n}=0$.

Lemma 2.6 [4] Let $\left\{a_{n}\right\}$ be a sequence of real numbers such that there exists a subsequence $\left\{n_{i}\right\}$ of $\{n\}$ such that $a_{n_{i}}<a_{n_{i}+1}$ for all $i \in \mathbb{N}$. Then there exists an increasing sequence $\left\{m_{k}\right\} \subset$ $\mathbb{N}$ such that $m_{k} \rightarrow \infty$ and the following properties are satisfied by all (sufficiently large) numbers $k \in \mathbb{N}$ :

$$
a_{m_{k}} \leq a_{m_{k}+1} \text { and } a_{k} \leq a_{m_{k}+1}
$$

In fact, $m_{k}$ is the largest number $n$ in the set $\{1,2, \ldots, k\}$ such that the condition $a_{n} \leq a_{n+1}$ holds.

Following the agreement in [26], we have the following lemma.

Lemma 2.7 Let $f: E \rightarrow(-\infty,+\infty]$ be a coercive Legendre function and $C$ be a nonempty, closed and convex subset of E. Let $A: C \rightarrow E^{*}$ be a continuous monotone mapping. For $r>0$ and $x \in E$, define the mapping $F_{r}: E \rightarrow C$ as follows:

$$
F_{r} x:=\left\{z \in C:\langle A z, y-z\rangle+\frac{1}{r}\langle\nabla f(z)-\nabla f(x), y-z\rangle \geq 0, \forall y \in C\right\}
$$

for all $x \in E$. Then the following hold:

(1) $F_{r}$ is single-valued;

(2) $F\left(F_{r}\right)=V I(C, A)$;

(3) $\phi\left(p, F_{r} x\right)+\phi\left(F_{r} x, x\right) \leq \phi(p, x)$ for $p \in F\left(F_{r}\right)$;

(4) $\operatorname{VI}(C, A)$ is closed and convex.

\section{Main result}

Let $C$ be a nonempty, closed and convex subset of $E$. Let $A_{i}: C \rightarrow E^{*}$, for $i=1,2, \ldots, N$, be continuous monotone mappings. For $r>0$, define $T_{i, r} x:=\left\{z \in C:\left\langle A_{i} z, y-z\right\rangle+\frac{1}{r}\langle\nabla f(z)-\right.$ 
$\nabla f(x), y-z\rangle \geq 0, \forall y \in C\}$ for all $x \in E$ and $i \in\{1,2, \ldots, N\}$, where $f$ is a Legendre and convex function from $E$ into $(-\infty,+\infty)$. Then, in what follows, we shall study the following iteration process:

$$
\left\{\begin{array}{l}
x_{0}=u \in C \quad \text { chosen arbitrarily, } \\
w_{n}=T_{N, r_{n}} \circ T_{N-1, r_{n}} \circ \cdots \circ T_{1, r_{n}} x_{n}, \\
x_{n+1}=P_{C}^{f} \nabla f^{*}\left(\alpha_{n} \nabla f(u)+\left(1-\alpha_{n}\right) \nabla f\left(w_{n}\right)\right), \quad \forall n \geq 0,
\end{array}\right.
$$

where $\left\{\alpha_{n}\right\} \subset(0,1)$ satisfies $\lim _{n \rightarrow \infty} \alpha_{n}=0$ and $\sum_{n=1}^{\infty}=\infty$, and $\left\{r_{n}\right\} \subset\left[c_{1}, \infty\right)$ for some $c_{1}>0$.

Theorem 3.1 Let $f: E \rightarrow \mathbb{R}$ be a strongly coercive Legendre function which is bounded, uniformly Fréchet differentiable and totally convex on bounded subsets of E. Let $C$ be a nonempty, closed and convex subset of $\operatorname{int}(\operatorname{dom} f)$ and $A_{i}: C \rightarrow E^{*}$, for $i=1,2, \ldots, N$, be a finite family of continuous monotone mappings with $\mathcal{F}:=\bigcap_{i=1}^{N} V I\left(C, A_{i}\right) \neq \emptyset$. Let $\left\{x_{n}\right\}_{n \geq 0}$ be a sequence defined by (3.1). Then $\left\{x_{n}\right\}$ converges strongly to $x^{*}=P_{\mathcal{F}}^{f}(u)$.

Proof By Lemma 2.7 we have that each $V I\left(C, A_{i}\right)$ for each $i \in\{1,2, \ldots, N\}$ and hence $\mathcal{F}$ are closed and convex. Thus, we can take $x^{*}:=P_{\mathcal{F}}^{f} u$. Let $u_{n, 1}=T_{1, r_{n}} x_{n}, u_{n, 2}=T_{2, r_{n}} u_{n, 1}$, $\ldots, u_{n, N-1}=T_{N-1, r_{n}} u_{n, N-2}$ and $u_{n, N}=T_{N, r_{n}} u_{n, N-1}=w_{n}$. Then, from (3.1), Lemmas 2.2, 2.3 and the property of $\phi$, we get that

$$
\begin{aligned}
D_{f}\left(x^{*}, x_{n+1}\right) & =D_{f}\left(x^{*}, P_{C}^{f} \nabla f^{*}\left(\alpha_{n} \nabla f(u)+\left(1-\alpha_{n}\right) \nabla f\left(w_{n}\right)\right)\right) \\
& \leq D_{f}\left(x^{*}, \nabla f^{*}\left(\alpha_{n} \nabla f(u)+\left(1-\alpha_{n}\right) \nabla f\left(w_{n}\right)\right)\right) \\
& \leq \alpha_{n} D_{f}\left(x^{*}, u\right)+\left(1-\alpha_{n}\right) D_{f}\left(x^{*}, w_{n}\right) \\
& =\alpha_{n} D_{f}\left(x^{*}, u\right)+\left(1-\alpha_{n}\right) D_{f}\left(x^{*}, T_{N, r_{n}} \circ T_{N-1, r_{n}} \circ \cdots \circ T_{1, r_{n}} x_{n}\right) \\
& \leq \alpha_{n} D_{f}\left(x^{*}, u\right)+\left(1-\alpha_{n}\right) D_{f}\left(x^{*}, x_{n}\right) .
\end{aligned}
$$

Thus, by induction,

$$
D_{f}\left(x^{*}, x_{n+1}\right) \leq \max \left\{D_{f}\left(x^{*}, x_{n}\right), D_{f}\left(x^{*}, u\right)\right\}, \quad \forall n \geq 0,
$$

which implies by Lemma 2.4 that $\left\{x_{n}\right\}$ and hence $\left\{w_{n}\right\}$ are bounded. Now let $z_{n}=$ $\nabla f^{*}\left(\alpha_{n} \nabla f(u)+\left(1-\alpha_{n}\right) \nabla f\left(w_{n}\right)\right)$. Then we have from (3.1) that $x_{n+1}=P_{C}^{f} z_{n}$. Using Lemmas 2.2, 2.3, 2.7(3), (2.3) and (2.4), we obtain that

$$
\begin{aligned}
D_{f}\left(x^{*}, x_{n+1}\right)= & D_{f}\left(x^{*}, P_{C}^{f} z_{n}\right) \leq D_{f}\left(x^{*}, z_{n}\right)=V\left(x^{*}, \nabla f\left(z_{n}\right)\right) \\
\leq & V\left(x^{*}, \nabla f\left(z_{n}\right)-\alpha_{n}\left(\nabla f(u)-\nabla f\left(x^{*}\right)\right)\right)+\left\langle\alpha_{n}\left(\nabla f(u)-\nabla f\left(x^{*}\right)\right), z_{n}-x^{*}\right\rangle \\
= & D_{f}\left(x^{*}, \nabla f^{*}\left(\alpha_{n} \nabla f\left(x^{*}\right)+\left(1-\alpha_{n}\right) \nabla f\left(w_{n}\right)\right)\right) \\
& +\alpha_{n}\left\langle\nabla f(u)-\nabla f\left(x^{*}\right), z_{n}-x^{*}\right\rangle \\
\leq & \alpha_{n} \phi\left(x^{*}, x^{*}\right)+\left(1-\alpha_{n}\right) D_{f}\left(x^{*}, w_{n}\right)+\alpha_{n}\left\langle\nabla f(u)-\nabla f\left(x^{*}\right), z_{n}-x^{*}\right\rangle \\
\leq & \left(1-\alpha_{n}\right) D_{f}\left(x^{*}, T_{N, r_{n}} u_{n, N-1}\right)+\alpha_{n}\left\langle\nabla f(u)-\nabla f\left(x^{*}\right), z_{n}-x^{*}\right\rangle,
\end{aligned}
$$


which implies that

$$
\begin{aligned}
D_{f}\left(x^{*}, x_{n+1}\right) \leq & \left(1-\alpha_{n}\right)\left[D_{f}\left(x^{*}, u_{n, N-1}\right)-D_{f}\left(w_{n}, u_{n, N-1}\right)\right] \\
& +\alpha_{n}\left\langle\nabla f(u)-\nabla f\left(x^{*}\right), z_{n}-x^{*}\right\rangle \\
\leq & \left(1-\alpha_{n}\right)\left[D_{f}\left(x^{*}, u_{n_{N}-2}\right)-D_{f}\left(u_{n_{N}-1}, u_{n_{N}-2}\right)\right] \\
& -\left(1-\alpha_{n}\right) D_{f}\left(w_{n}, u_{n, N-1}\right)+\alpha_{n}\left\langle\nabla f(u)-\nabla f\left(x^{*}\right), z_{n}-x^{*}\right\rangle \\
& \cdots \\
\leq & \left(1-\alpha_{n}\right) D_{f}\left(x^{*}, x_{n}\right)-\left(1-\alpha_{n}\right)\left[D_{f}\left(u_{n, 1}, x_{n}\right)+D_{f}\left(u_{n, 2}, u_{n, 1}\right)\right. \\
& \left.+\cdots+D_{f}\left(u_{n, N-1}, u_{n, N-2}\right)+D_{f}\left(w_{n}, u_{n, N-1}\right)\right] \\
& +\alpha_{n}\left\langle\nabla f(u)-\nabla f\left(x^{*}\right), z_{n}-x^{*}\right\rangle \\
\leq & \left(1-\alpha_{n}\right) D_{f}\left(x^{*}, x_{n}\right)+\alpha_{n}\left\langle\nabla f(u)-\nabla f\left(x^{*}\right), z_{n}-x^{*}\right\rangle .
\end{aligned}
$$

Now, we consider two possible cases.

Case 1 . Suppose that there exists $n_{0} \in N$ such that $\left\{D_{f}\left(x^{*}, x_{n}\right)\right\}$ is decreasing. Then we obtain that $\left\{D_{f}\left(x^{*}, x_{n}\right)\right\}$ is convergent. Thus, from (3.3) we have that $D_{f}\left(u_{n, 1}, x_{n}\right), D_{f}\left(u_{n, 2}, u_{n, 1}\right)$, $\ldots, D_{f}\left(w_{n}, u_{n, N-1}\right) \rightarrow 0$ as $n \rightarrow \infty$, and hence by Lemma 2.1 we get that

$$
u_{n, 1}-x_{n} \rightarrow 0, \quad u_{n, 2}-u_{n, 1} \rightarrow 0, \quad \ldots, \quad w_{n}-u_{n, N-1} \rightarrow 0 \quad \text { as } n \rightarrow \infty .
$$

Furthermore, from the property of $D_{f}(\cdot, \cdot)$ and the fact that $\alpha_{n} \rightarrow 0$ as $n \rightarrow \infty$, we have that

$$
\begin{aligned}
D_{f}\left(w_{n}, z_{n}\right) & =D_{f}\left(w_{n}, \nabla f^{*}\left(\alpha_{n} \nabla f(u)+\left(1-\alpha_{n}\right) \nabla f\left(w_{n}\right)\right)\right) \\
& \leq \alpha_{n} D_{f}\left(w_{n}, u\right)+\left(1-\alpha_{n}\right) D_{f}\left(w_{n}, w_{n}\right) \\
& \leq \alpha_{n} D_{f}\left(w_{n}, u\right)+\left(1-\alpha_{n}\right) D_{f}\left(w_{n}, w_{n}\right) \rightarrow 0 \text { as } n \rightarrow \infty,
\end{aligned}
$$

and hence from Lemma 2.1 we have that $w_{n}-z_{n} \rightarrow 0$ and this with (3.4) implies that

$$
z_{n}-u_{n, N-1} \rightarrow 0, \quad z_{n}-u_{n, N-2} \rightarrow 0, \quad \ldots, \quad z_{n}-u_{n, 1} \rightarrow 0 \quad \text { as } n \rightarrow \infty .
$$

Since $\left\{z_{n}\right\}$ is bounded and $E$ is reflexive, we choose a subsequence $\left\{z_{n_{k}}\right\}$ of $\left\{z_{n}\right\}$ such that $z_{n_{k}} \rightarrow z$ and $\lim \sup _{n \rightarrow \infty}\left\langle\nabla f(u)-\nabla f\left(x^{*}\right), z_{n}-x^{*}\right\rangle=\lim _{k \rightarrow \infty}\left\langle\nabla f(u)-\nabla f\left(x^{*}\right), z_{n_{k}}-x^{*}\right\rangle$. Then, from (3.5) and (3.4), we get that $u_{n_{k}, i} \rightarrow z$ for each $i \in\{1,2, \ldots, N\}$.

Now, we show that $z \in \operatorname{VI}\left(C, A_{i}\right)$ for each $i \in\{1,2, \ldots, N\}$. But from the definition of $u_{n, i}$, we have that

$$
\left\langle A_{i} u_{n, i}, y-u_{n, i}\right\rangle+\left\langle\frac{\nabla f\left(u_{n, i}\right)-\nabla f\left(x_{n}\right)}{r_{n}}, y-u_{n, i}\right\rangle \geq 0, \quad \forall y \in C,
$$

and hence

$$
\left\langle A_{i} u_{n_{k}, i}, y-u_{n_{k}, i}\right\rangle+\left\langle\frac{\nabla f\left(u_{n_{k}, i}\right)-\nabla f\left(x_{n_{k}}\right)}{r_{n_{k}}}, y-u_{n_{k}, i}\right\rangle \geq 0, \quad \forall y \in C
$$


for each $i \in\{1,2, \ldots, N\}$. Set $v_{t}=t y+(1-t) z$ for all $t \in(0,1]$ and $y \in C$. Consequently, we get that $v_{t} \in C$. Now, from (3.6) it follows that

$$
\begin{aligned}
\left\langle A_{i} v_{t}, v_{t}-u_{n_{k}, i}\right\rangle \geq & \left\langle A_{i} v_{t}, v_{t}-u_{n_{k}, i}\right\rangle-\left\langle A_{i} u_{n_{k}, i}, v_{t}-u_{n_{k}, i}\right\rangle \\
& -\left\langle\frac{\nabla f\left(u_{n_{k}, i}\right)-\nabla f\left(x_{n_{k}}\right)}{r_{n_{k}}}, v_{t}-u_{n_{k}, i}\right\rangle \\
= & \left\langle A_{i} v_{t}-A_{i} u_{n_{k}, i}, v_{t}-u_{n_{k}, i}\right\rangle \\
& -\left\langle\frac{\nabla f\left(u_{n_{k}, i}\right)-\nabla f\left(x_{n_{k}}\right)}{r_{n_{k}}}, v_{t}-u_{n_{k}, i}\right\rangle .
\end{aligned}
$$

In addition, since $f$ is uniformly Fréchet differentiable and bounded, we have that $\nabla f$ is uniformly continuous (see [36]). Thus, from (3.4) and the uniform continuity of $\nabla f$, we obtain that

$$
\frac{\nabla f\left(u_{n_{k}, i}\right)-\nabla f\left(x_{n_{k}}\right)}{r_{n_{k}}} \rightarrow 0 \quad \text { as } k \rightarrow \infty
$$

and since $A$ is monotone, we also have that $\left\langle A_{i} v_{t}-A_{i} u_{n_{k}, i}, v_{t}-u_{n_{k}, i}\right\rangle \geq 0$. Thus, it follows that

$$
0 \leq \lim _{k \rightarrow \infty}\left\langle A_{i} v_{t}, v_{t}-u_{n_{k}, i}\right\rangle=\left\langle A_{i} v_{t}, v_{t}-z\right\rangle
$$

and hence

$$
\left\langle A_{i} v_{t}, y-z\right\rangle \geq 0, \quad \forall y \in C \text {, for all } i \in\{1,2, \ldots, N\} .
$$

If $t \rightarrow 0$, the continuity of $A_{i}$ implies that

$$
\left\langle A_{i} z, y-z\right\rangle \geq 0, \quad \forall y \in C
$$

This implies that $z \in V I\left(C, A_{i}\right)$ for all $i \in\{1,2, \ldots, N\}$.

Therefore, we obtain that $z \in \bigcap_{i=1}^{N} V I\left(C, A_{i}\right)$. Thus, by Lemma 2.2, we immediately obtain that $\lim _{\sup _{n \rightarrow \infty}}\left\langle\nabla f(u)-\nabla f\left(x^{*}\right), z_{n}-x^{*}\right\rangle=\left\langle\nabla f(u)-\nabla f\left(x^{*}\right), z-x^{*}\right\rangle \leq 0$. It follows from Lemma 2.5 and (3.3) that $D_{f}\left(x^{*}, x_{n}\right) \rightarrow 0$ as $n \rightarrow \infty$. Consequently, $x_{n} \rightarrow x^{*}$.

Case 2. Suppose that there exists a subsequence $\left\{n_{j}\right\}$ of $\{n\}$ such that

$$
D_{f}\left(x^{*}, x_{n_{j}}\right)<D_{f}\left(x^{*}, x_{n_{j}+1}\right)
$$

for all $j \in \mathbb{N}$. Then, by Lemma 2.6, there exists a nondecreasing sequence $\left\{m_{k}\right\} \subset \mathbb{N}$ such that $m_{k} \rightarrow \infty, D_{f}\left(x^{*}, x_{m_{k}}\right) \leq D_{f}\left(x^{*}, x_{m_{k}+1}\right)$ and $D_{f}\left(x^{*}, x_{k}\right) \leq D_{f}\left(x^{*}, x_{m_{k}+1}\right)$ for all $k \in \mathbb{N}$. From (3.3) and $\alpha_{n} \rightarrow 0$, we have

$$
\begin{aligned}
& \left(1-\alpha_{m_{k}}\right)\left(D_{f}\left(u_{m_{k}, 1}, x_{m_{k}}\right)+\cdots+D_{f}\left(w_{m_{k}}, u_{m_{k}, N-1}\right)\right) \\
& \leq\left(D_{f}\left(x^{*}, x_{m_{k}}\right)-D_{f}\left(x^{*}, x_{m_{k}+1}\right)\right)+\alpha_{m_{k}} D_{f}\left(x^{*}, x_{m_{k}}\right) \\
& \quad+\alpha_{m_{k}}\left\langle\nabla f(u)-\nabla f\left(x^{*}\right), z_{m_{k}}-x^{*}\right\rangle \rightarrow 0 \text { as } k \rightarrow \infty,
\end{aligned}
$$


which implies that $D_{f}\left(u_{m_{k}, 1}, x_{m_{k}}\right), \ldots, D_{f}\left(w_{m_{k}}, u_{m_{k}, N-1}\right) \rightarrow 0$, and hence $u_{m_{k}, 1}-x_{m_{k}} \rightarrow$ $0, \ldots, w_{m_{k}}-u_{m_{k}, N-1} \rightarrow 0$ as $k \rightarrow \infty$. Thus, as in Case 1, we obtain that

$$
\limsup _{k \rightarrow \infty}\left\langle\nabla f(u)-\nabla f\left(x^{*}\right), z_{m_{k}}-x^{*}\right\rangle \leq 0
$$

Furthermore, from (3.3) we have that

$$
D_{f}\left(x^{*}, x_{m_{k}+1}\right) \leq\left(1-\alpha_{m_{k}}\right) D_{f}\left(x^{*}, x_{m_{k}}\right)+\alpha_{m_{k}}\left\langle\nabla f(u)-\nabla f\left(x^{*}\right), z_{m_{k}}-x^{*}\right\rangle .
$$

Thus, since $D_{f}\left(x^{*}, x_{m_{k}}\right) \leq D_{f}\left(x^{*}, x_{m_{k}+1}\right)$, we get that

$$
\begin{aligned}
\alpha_{m_{k}} D_{f}\left(x^{*}, x_{m_{k}}\right) \leq & D_{f}\left(x^{*}, x_{m_{k}}\right)-D_{f}\left(x^{*}, x_{m_{k}+1}\right) \\
& +\alpha_{m_{k}}\left\langle\nabla f(u)-\nabla f\left(x^{*}\right), z_{m_{k}}-x^{*}\right\rangle \\
\leq & \alpha_{m_{k}}\left\langle\nabla f(u)-\nabla f\left(x^{*}\right), z_{m_{k}}-x^{*}\right\rangle .
\end{aligned}
$$

Moreover, since $\alpha_{m_{k}}>0$, we obtain that

$$
D_{f}\left(x^{*}, x_{m_{k}}\right) \leq\left\langle\nabla f(u)-\nabla f\left(x^{*}\right), z_{m_{k}}-x^{*}\right\rangle .
$$

It follows from (3.7) that $D_{f}\left(x^{*}, x_{m_{k}}\right) \rightarrow 0$ as $k \rightarrow \infty$. This together with (3.8) implies that $D_{f}\left(x^{*}, x_{m_{k}+1}\right) \rightarrow 0$. Therefore, since $D_{f}\left(x^{*}, x_{k}\right) \leq D_{f}\left(x^{*}, x_{m_{k}+1}\right)$ for all $k \in \mathbb{N}$, we conclude that $x_{k} \rightarrow x^{*}$ as $k \rightarrow \infty$. Hence, both cases imply that $\left\{x_{n}\right\}$ converges strongly to $x^{*}=P_{F}^{f} u$ and the proof is complete.

If in Theorem $3.1 N=1$, then we get the following corollary.

Corollary 3.2 Let $f: E \rightarrow \mathbb{R}$ be a strongly coercive Legendre function which is bounded, uniformly Fréchet differentiable and totally convex on bounded subsets of E. Let $C$ be a nonempty, closed and convex subset of $\operatorname{int}(\operatorname{dom} f)$, and let $A: C \rightarrow E^{*}$ be a continuous monotone mapping with $\operatorname{VI}(C, A) \neq \emptyset$. Let $\left\{x_{n}\right\}_{n \geq 0}$ be a sequence defined by (3.1),

$$
\left\{\begin{array}{l}
x_{0}=u \in C \quad \text { chosen arbitrarily, } \\
w_{n}=T_{r_{n}} x_{n}, \\
x_{n+1}=P_{C}^{f} \nabla f^{*}\left(\alpha_{n} \nabla f(u)+\left(1-\alpha_{n}\right) \nabla f\left(w_{n}\right)\right),
\end{array}\right.
$$

where $T_{\gamma} x:=\left\{z \in C:\langle A z, y-z\rangle+\frac{1}{\gamma}\langle\nabla f(z)-\nabla f(x), y-z\rangle \geq 0, \forall y \in C\right\}$ for all $x \in E ; \alpha_{n} \in$ $(0,1)$ satisfies $\lim _{n \rightarrow \infty} \alpha_{n}=0$ and $\sum_{n=1}^{\infty} \alpha_{n}=\infty$ and $\left\{r_{n}\right\} \subset\left[c_{1}, \infty\right)$ for some $c_{1}>0$. Then the sequence $\left\{x_{n}\right\}_{n \geq 0}$ converges strongly to a point $x^{*}=P_{V I(C, A)}(u)$.

If $C=E$, then $V I(C, A)=A^{-1}(0)$ and hence the following corollary holds.

Corollary 3.3 Let $f: E \rightarrow \mathbb{R}$ be a strongly coercive Legendre function which is bounded, uniformly Fréchet differentiable and totally convex on bounded subsets of E. Let $A_{i}$ : $E \rightarrow E^{*}$, for $i=1,2, \ldots, N$, be a finite family of continuous monotone mappings. Let $\mathcal{F}:=$ $\bigcap_{i=1}^{N} V I\left(C, A_{i}\right)=\bigcap_{i=1}^{N} A^{-1}(0) \neq \emptyset$. Let $\left\{x_{n}\right\}_{n \geq 0}$ be a sequence defined by (3.1). Then $\left\{x_{n}\right\}$ converges strongly to $x^{*}=P_{\mathcal{F}}^{f}(u)$. 
If in Theorem 3.1 we assume $u=0$, then the scheme converges strongly to the common minimum-norm zero of a finite family of continuous monotone mappings. In fact, we have the following corollary.

Corollary 3.4 Let $f: E \rightarrow \mathbb{R}$ be a strongly coercive Legendre function which is bounded, uniformly Fréchet differentiable and totally convex on bounded subsets of $E$. Let $C$ be a nonempty, closed and convex subset of $\operatorname{int}(\operatorname{dom} f)$, and let $A_{i}: C \rightarrow E^{*}$, for $i=1,2, \ldots, N$, be a finite family of continuous monotone mappings with $\mathcal{F}:=\bigcap_{i=1}^{N} V I\left(C, A_{i}\right) \neq \emptyset$. Let $\left\{x_{n}\right\}_{n \geq 0}$ be a sequence defined by (3.1) with $u=0$. Then $\left\{x_{n}\right\}$ converges strongly to $x^{*}=P_{\mathcal{F}}^{f}(0)$, which is the common minimum-norm (with respect to the Bregman distance) solution of the variational inequalities.

\section{Application}

In this section, we study the problem of finding a minimizer of a continuously Fréchet differentiable convex functional in Banach spaces.

Let $g_{i}$, for $i=1,2, \ldots, N$, be continuously Fréchet differentiable convex functionals such that the gradients of $g_{i},\left.\left(\nabla g_{i}\right)\right|_{C}$ are continuous and monotone. For $r>0$, let $K_{i, r} x:=\{z \in C$ : $\left.\left\langle\nabla g_{i}(z), y-z\right\rangle+\frac{1}{r}\langle\nabla f(z)-\nabla f(x), y-z\rangle \geq 0, \forall y \in C\right\}$ for all $x \in E$ and for each $i \in\{1,2, \ldots, N\}$. Then the following theorem holds.

Theorem 4.1 Let $f: E \rightarrow \mathbb{R}$ be a strongly coercive Legendre function which is bounded, uniformly Fréchet differentiable and totally convex on bounded subsets of E. Let $g_{i}, i=$ $1,2, \ldots, N$, be continuously Fréchet differentiable convex functionals such that the gradients of $g_{i},\left.\left(\nabla g_{i}\right)\right|_{C}$ are continuous, monotone and $\mathcal{F}:=\bigcap_{i=1}^{N} \arg \min _{y \in C} g_{i}(y) \neq \emptyset$, where $\arg \min _{y \in C} g_{i}(y):=\left\{z \in C: g_{i}(z)=\min _{y \in C} g_{i}(y)\right\}$. Let $\left\{x_{n}\right\}_{n \geq 0}$ be a sequence defined by

$$
\left\{\begin{array}{l}
x_{0}=u \in C \quad \text { chosen arbitrarily, } \\
w_{n}=K_{N, r_{n}} \circ K_{N-1, r_{n}} \circ \cdots \circ K_{1, r_{n}} x_{n}, \\
x_{n+1}=P_{C}^{f} \nabla f^{*}\left(\alpha_{n} \nabla f(u)+\left(1-\alpha_{n}\right) \nabla f\left(w_{n}\right)\right), \quad \forall n \geq 0,
\end{array}\right.
$$

where $\alpha_{n} \in(0,1)$ satisfies $\lim _{n \rightarrow \infty} \alpha_{n}=0$ and $\sum_{n=1}^{\infty} \alpha_{n}=\infty$ and $\left\{r_{n}\right\} \subset\left[c_{1}, \infty\right)$ for some $c_{1}>0$. Then the sequence $\left\{x_{n}\right\}$ converges strongly to $p=P_{\mathcal{F}}^{f}(u)$.

Proof We note that from the convexity and Fréchet differentiability of $f$, we have $V I\left(C,\left.\left(\nabla g_{i}\right)\right|_{C}\right)=\arg \min _{y \in C} g_{i}(y)$ for each $i \in\{1,2, \ldots, N\}$. Thus, by Theorem 3.1, $\left\{x_{n}\right\}$ converges strongly to $p=P_{\mathcal{F}}^{f}(u)$.

Remark 4.2 Our results are new even if the convex function $f$ is chosen to be $f(x)=\frac{1}{p}\|x\|^{p}$ $(1<p<\infty)$ in uniformly smooth and uniformly convex spaces.

Remark 4.3 Our theorems extend and unify most of the results that have been proved for this important class of nonlinear operators. In particular, Theorem 3.1 extends Theorem 3.3 of [16], Theorem 3.1 of [17], Theorem 3.1 of [17] and Theorem 3.3 of [10] and Theorem 4.2 of [25] either to a more general class of continuous monotone operators or to a more general Banach space $E$. Moreover, in all our theorems and corollaries, the computation of $C_{n}$ or $Q_{n}$ for each $n \geq 1$ is not required. 


\section{Competing interests}

The authors declare that they have no competing interests.

\section{Authors' contributions}

All authors contributed equally to this work. All authors read and approved final manuscript.

\section{Author details}

${ }^{1}$ Department of Mathematics, King Abdulaziz University, P.O. Box 80203, Jeddah, 21589, Saudi Arabia. ${ }^{2}$ Department of Mathematics, University of Botswana, Pvt. Bag 00704, Gaborone, Botswana.

\section{Acknowledgements}

This article was funded by the Deanship of Scientific Research (DSR), King Abdulaziz University, Jeddah. The first and third authors acknowledge with thanks DSR for financial support. The second author undertook this work when he was visiting the Abdus Salam International Center for Theoretical Physics (ICTP), Trieste, Italy, as a regular associate.

Received: 27 August 2013 Accepted: 14 November 2013 Published: 13 Dec 2013

\section{References}

1. Bregman, LM: The relaxation method for finding the common point of convex sets and its application to the solution of problems in convex programming. USSR Comput. Math. Math. Phys. 7, 200-217 (1967)

2. Alber, Yl: Metric and generalized projection operators in Banach spaces: properties and applications. In: Theory and Applications of Nonlinear Operators of Accretive and Monotone Type. Lect. Notes Pure Appl. Math., pp. 15-50 (1996)

3. Kamimura, S, Takahashi, W: Strong convergence of proximal-type algorithm in a Banach space. SIAM J. Optim. 13, 938-945 (2002)

4. Maingé, PE: Strong convergence of projected subgradient methods for nonsmooth and non-strictly convex minimization. Set-Valued Anal. 16, 899-912 (2008)

5. Zegeye, H, Ofoedu, EU, Shahzad, N: Convergence theorems for equilibrium problem, variational inequality problem and countably infinite relatively quasi-nonexpansive mappings. Appl. Math. Comput. 216, 3439-3449 (2010)

6. Zegeye, $\mathrm{H}$, Shahzad, N: Strong convergence theorems for a finite family of nonexpansive mappings and semi-groups via the hybrid method. Nonlinear Anal. 72, 325-329 (2010)

7. Zegeye, $\mathrm{H}$, Shahzad, N: A hybrid approximation method for equilibrium, variational inequality and fixed point problems. Nonlinear Anal. Hybrid Syst. 4, 619-630 (2010)

8. Zegeye, $\mathrm{H}$, Shahzad, $\mathrm{N}$ : A hybrid scheme for finite families of equilibrium, variational inequality and fixed point problems. Nonlinear Anal. 74, 263-272 (2011)

9. Zegeye, $\mathrm{H}$, Shahzad, N: Convergence theorems for a common point of solutions of equilibrium and fixed point of relatively nonexpansive multi-valued mapping problems. Abstr. Appl. Anal. 2012, Article ID 859598 (2012)

10. liduka, H, Takahashi, W: Strong convergence studied by a hybrid type method for monotone operators in a Banach space. Nonlinear Anal. 68, 3679-3688 (2008)

11. Kinderlehrer, D, Stampaccia, G: An Iteration to Variational Inequalities and Their Applications. Academic Press, New York (1990)

12. Lions, JL, Stampacchia, G: Variational inequalities. Commun. Pure Appl. Math. 20, 493-517 (1967)

13. Zegeye, $\mathrm{H}$, Shahzad, $\mathrm{N}$ : Strong convergence for monotone mappings and relatively weak nonexpansive mappings. Nonlinear Anal. 70, 2707-2716 (2009)

14. Zegeye, H, Shahzad, N, Alghamdi, MA: Strong convergence theorems for a common point of solution of variational inequality, solutions of equilibrium and fixed point problems. Fixed Point Theory Appl. 2012, 119 (2012)

15. Zegeye, $\mathrm{H}$, Shahzad, $\mathrm{N}$ : An iteration to a common point of solution of variational inequality and fixed point-problems in Banach spaces. J. Appl. Math. 2012, Article ID 504503 (2012)

16. liduka, H, Takahashi, W, Toyoda, M: Approximation of solutions of variational inequalities for monotone mappings. Panam. Math. J. 14, 49-61 (2004)

17. liduka, $\mathrm{H}$, Takahashi, W: Weak convergence of projection algorithm for variational inequalities in Banach spaces J. Math. Anal. Appl. 339, 668-679 (2008)

18. Zegeye, $\mathrm{H}$, Shahzad, N: Approximating common solution of variational inequality problems for two monotone mappings in Banach spaces. Optim. Lett. 5, 691-704 (2011)

19. Bauschke, HH, Borwein, JM, Combettes, PL: Essential smoothness, essential strict convexity, and Legendre functions in Banach spaces. Commun. Contemp. Math. 3, 615-647 (2001)

20. Bauschke, HH, Borwein, JM, Combettes, PL: Bregman monotone optimization algorithms. SIAM J. Control Optim. 42, 596-636 (2003)

21. Butnariu, D, lusem, AN, Zalinescu, C: On uniform convexity, total convexity and convergence of the proximal point and outer Bregman projection algorithms in Banach spaces. J. Convex Anal. 10, 35-61 (2003)

22. Butnariu, D, Resmerita, E: Bregman distances, totally convex functions and a method for solving operator equations in Banach spaces. Abstr. Appl. Anal. 2006, Article ID 84919 (2006)

23. Reich, S: A weak convergence theorem for the alternating method with Bregman distances. In: Theory and Applications of Nonlinear Operators of Accretive and Monotone Type, pp. 313-318. Dekker, New York (1996)

24. Reich, S, Sabach, S: A projection method for solving nonlinear problems in reflexive Banach spaces. J. Fixed Point Theory Appl. 9, 101-116 (2011)

25. Reich, S, Sabach, S: Two strong convergence theorems for a proximal method in reflexive Banach spaces. Numer. Funct. Anal. Optim. 31, 22-44 (2010)

26. Reich, S, Sabach, S: Two strong convergence theorems for Bregman strongly nonexpansive operators in reflexive Banach spaces. Nonlinear Anal. TMA 73, 122-135 (2010)

27. Reich, S, Sabach, S: Existence and approximation of fixed points of Bregman firmly nonexpansive mappings in reflexive Banach spaces. In: Fixed-Point Algorithms for Inverse Problems in Science and Engineering, pp. 301-316. Springer, New York (2011) 
28. Bonnans, JF, Shapiro, A: Perturbation Analysis of Optimization Problems. Springer, New York (2000)

29. Phelps, RP: Convex Functions, Monotone Operators, and Differentiability, 2nd edn. Lecture Notes in Mathematics, vol. 1364. Springer, Berlin (1993)

30. Hiriart-Urruty, J-B, Lemarchal, C: Convex Analysis and Minimization Algorithms II. Grundlehren der Mathematischen Wissenschaften, vol. 306. Springer, Berlin (1993)

31. Butnariu, D, lusem, AN: Totally Convex Functions for Fixed Points Computation and Infinite Dimensional Optimization. Kluwer Academic, Dordrecht (2000)

32. Martin-Marquez, V, Reich, S, Sabach, S: Bregman strongly nonexpansive operators in reflexive Banach spaces. J. Math. Anal. Appl. 400, 597-614 (2013)

33. Censor, Y, Lent, A: An iterative row-action method for interval convex programming. J. Optim. Theory Appl. 34 , $321-353(1981)$

34. Kohsaka, F, Takahashi, W: Proximal point algorithms with Bregman functions in Banach spaces. J. Nonlinear Convex Anal. 6, 505-523 (2005)

35. Xu, HK: Another control condition in an iterative method for nonexpansive mappings. Bull. Aust. Math. Soc. 65 109-113 (2002)

36. Reich, S, Sabach, S: A strong convergence theorem for a proximal-type algorithm in reflexive Banach spaces. J. Nonlinear Convex Anal. 10, 471-485 (2009)

10.1186/1687-1812-2013-343

Cite this article as: Shahzad et al.: Convergence results for a common solution of a finite family of variational inequality problems for monotone mappings with Bregman distance function. Fixed Point Theory and Applications 2013, 2013:343

\section{Submit your manuscript to a SpringerOpen ${ }^{\circ}$ journal and benefit from:}

- Convenient online submission

- Rigorous peer review

- Immediate publication on acceptance

- Open access: articles freely available online

- High visibility within the field

- Retaining the copyright to your article 